\title{
Analysis of the Raman Frequency Shifts for the Lattice Modes and Vibrons Related to the Thermodynamic Quantities in the $\eta$ Phase of Solid Nitrogen
}

\begin{abstract}
The thermodynamic quantities of the isothermal compressibility, thermal expansion and the specific heat are calculated here as a function of pressure by using the observed Raman frequencies of the lattice modes and vibrons in the $\eta$ phase of solid nitrogen. The Pippard relations and their spectroscopic modifications are constructed, and the slope $\mathrm{dP} / \mathrm{dT}$ is deduced from the Raman frequency shifts in this phase of $\mathrm{N}_{2}$. It is shown that the thermodynamic quantities can be predicted from the Raman frequency shifts, in particular, in the $\eta$ phase of solid nitrogen.
\end{abstract}

Keywords: Raman frequency, thermodynamic quantities, $\eta$ phase, solid nitrogen

PACS $^{\circledast}$ (2010). 64.10.+h, 64.60.-i, 64.70.-p

*Corresponding author: Hamit Yurtseven: Department of Physics, Middle East Technical University, Ankara 06531, Turkey E-mail: hamit@metu.edu.tr

Özge Akay: Department of Physics, Middle East Technical University, Ankara 06531, Turkey; Department of Physics, Bilkent University, Ankara 06800, Turkey

\section{Introduction}

The existence of the solid phase $\eta-\mathrm{N}_{2}$ has been reported in the literature from a Raman experiment when the lowerfrequency vibron splits at a pressure of $20 \mathrm{GPa}$ at room temperature [1], as also pointed out in a previous work [2]. Close to the $\eta$ phase, it has also been observed from the Raman scattering that the vibrational frequency shifts exhibit an anomalous behavior with decreasing temperature in the $\delta$ phase of nitrogen [3]. This has been interpreted as the transition due to changes in the orientational behavior of the $\mathrm{N}_{2}$ molecules from a free rotation into an orientationally localized mode at $10.5 \mathrm{GPa}$ at room tem- perature (300 K) [4]. An evidence has been found from the high-pressure Raman scattering for a dynamical freezing of the $\mathrm{N}_{2}$ molecules within the $\delta$ phase and the $\delta$ - $\epsilon$ transition has been observed by a drop of the vibrational linewidths around $17 \mathrm{GPa}$ [4]. The Raman experiments have determined that the $\eta$ phase is the nonmolecular phase which occurs at around $135 \mathrm{GPa}$ in the solid nitrogen [5]. The experimental P-T phase diagram of $\mathrm{N}_{2}$ as given in a previous work [2], has been constructed using the experimental data [1], [2], [6-9]. It includes the phases of $€, \delta, \zeta$ and $\eta$, and also the low temperature-low pressure phases of $\alpha, \beta$ and $\gamma$ of solid $N_{2}$. The $\alpha, \beta$ and $\gamma$ phases have been studied extensively by using various experimental techniques, mainly the Raman spectroscopy [2], [4], [5], [10-12] and x-ray diffraction [1], [5], [9]. We have also studied recently the temperature dependence of the Raman frequencies of the lattice and internal modes for the $\gamma$ and $\beta$ phases [13]. Some theoretical studies on the $\alpha, \beta$ and $\gamma$ phases have also been reported in the literature [13-17]. Thermodynamic properties of the solid nitrogen have also been studied for various phases. Studies on the thermal expansivity [18], [19] and the specific heat [20] have been given in the literature. The volume dependence of the Raman frequencies of the lattice and internal modes through the mode Grüneisen parameter has been studied extensively in the $\alpha, \beta$ and $\gamma$ phases of $\mathrm{N}_{2}$ [12].

In this study, we relate the Raman frequency shifts for the lattice (external) modes and vibrons (internal modes) of $\mathrm{N}_{2}$ to the thermodynamic quantities such as the isothermal compressibility $\kappa_{\mathrm{T}}$, thermal expansion $\alpha_{\mathrm{p}}$ and the specific heat $\mathrm{C}_{\mathrm{p}}-\mathrm{C}_{\mathrm{v}}$ for the $\eta$ phase of solid $\mathrm{N}_{2}$. By using the experimental data [2] for the pressure dependence of the lattice modes $\left(v_{L 1}^{\prime}\right.$ and $\left.v_{L 2}^{\prime}\right)$ and internal modes $\left(v_{1}\right.$ and $\left.v_{2(2)}\right)$, the critical behavior of the isothermal compressibility $\kappa_{T}$ close to the $\eta-\delta$ transition is investigated. The pressure dependences of $\alpha_{p}$ and $C_{p}-C_{v}$ are then calculated. The spectroscopic modification of the Pippard relation, $\alpha_{p}$ vs. $(1 / \mathrm{v})(\mathrm{av} / \mathrm{aP})_{\mathrm{T}}$, for the lattice and internal modes is examined in the $\eta$ phase of solid nitrogen. Also, the Pippard relation of $\mathrm{C}_{\mathrm{p}}-\mathrm{C}_{\mathrm{v}}$ vs. $\alpha_{\mathrm{p}}$ is calculated from the frequency 


\begin{tabular}{lllllll} 
Raman modes & $\mathbf{v}_{\mathbf{0}}(\mathbf{c m})^{-1}$ & $\mathbf{c}^{\prime}\left(\mathbf{c m}^{-1} / \mathbf{G P a}^{2}\right)$ & $\mathbf{c}^{\prime}$ & $\mathbf{c}_{\mathbf{0}}$ & $\mathbf{c}_{\mathbf{1}} \times 10^{-2}(\mathbf{G P a})^{-1}$ & $\mathbf{c}_{\mathbf{2}} \times 10^{-4}(\mathrm{GPa})^{-2}$ \\
\hline $\mathrm{U}_{\mathrm{L} 1}^{\prime}$ & 276.31 & 5.22 & - & 1.1 & 2.36 & 3.0 \\
$\mathrm{U}_{\mathrm{L} 2}^{\prime}$ & 179.43 & 4.35 & - & 2.1 & 3.19 & 5.0 \\
$\mathrm{U}_{1}$ & 2332.9 & 3.5 & -0.024 & 0.05 & 0.17 & 1.0 \\
$\mathrm{U}_{2 \mathrm{C}(2)}$ & 2325.2 & 2.93 & -0.027 & 0.01 & 0.33 & 0.5 \\
\hline
\end{tabular}

Table 1: Values of the coefficients $v_{0}$ and $c$ for the pressure shifts of the $N_{2}$ lattice mode vibrons $\left(v_{L_{1}}^{\prime}\right.$ and $\left.v_{L_{2}}^{\prime}\right)$ (Eq. 1) and for the internal modes $\left(v_{1}\right.$ and $v_{2 c(2)}$ ) (Eq. 2). Values of the coefficients $c_{0}, c_{1}$ and $c_{2}$ for the Grüneisen parameter $\gamma_{T}$ of the Raman modes indicated according to Eq. (3), are also given here.

shifts of those Raman modes is constructed for the $\eta$ phase of solid $\mathrm{N}_{2}$.

Below, in section 2 we give our analysis and results. Discussion and conclusions are given in sections 3 and 4, respectively.

\section{Calculations and results}

The pressure dependences of the Raman frequency shifts for the lattice modes and vibrons were analyzed and they were related to the thermodynamic quantities of the isothermal compressibility $\kappa_{\mathrm{T}}$, thermal expansion $\alpha_{\mathrm{p}}$ and the specific heat $\left(C_{p}-C_{v}\right)$ in the $\eta$ phase of solid nitrogen.

\subsection{Raman frequency shifts of the lattice modes and $\mathrm{N}_{2}$ vibrons}

The Raman frequencies for the lattice modes of $\mathrm{v}_{\mathrm{L} 1}^{\prime}$ and $v^{\prime}{ }_{\mathrm{L} 2}$ which were measured at various pressures $(\mathrm{T}=300 \mathrm{~K})$ [2], were analyzed for the $\eta$ phase of $\mathrm{N}_{2}$ using

$$
v=v_{0}+c P
$$

where $v_{0}$ and $c$ are constants. For this analysis, the coefficients $v_{0}$ and $c$ were determined as given in Table 1. Fig. 1 gives the Raman frequencies plotted as a function of pressure $(\mathrm{T}=300 \mathrm{~K})$ for the lattice modes $\left(\mathrm{v}_{\mathrm{L} 1}^{\prime}\right.$ and $\left.\mathrm{v}_{\mathrm{L} 2}^{\prime}\right)$. Solid lines in this figure represent Eq. (1) fitted to the experimental data [2].

For the internal modes of $v_{1}$ and $v_{2(2)}$, a quadratic polynomial fit was employed to the experimental data [2] according to

$$
\mathrm{v}=\mathrm{v}_{0}+\mathrm{cP}+\mathrm{c}^{\prime} \mathrm{P}^{2}
$$

where $v_{0}, c$ and $c^{\prime}$ are constants. Our fit gave us the values of the coefficients, as given in Table 1. Fig. 2 gives a plot of Raman frequency as a function of pressure for the internal modes $\left(v_{1}\right.$ and $\left.v_{2 c(2)}\right)$ in the $\eta$ phase of solid $N_{2}$.

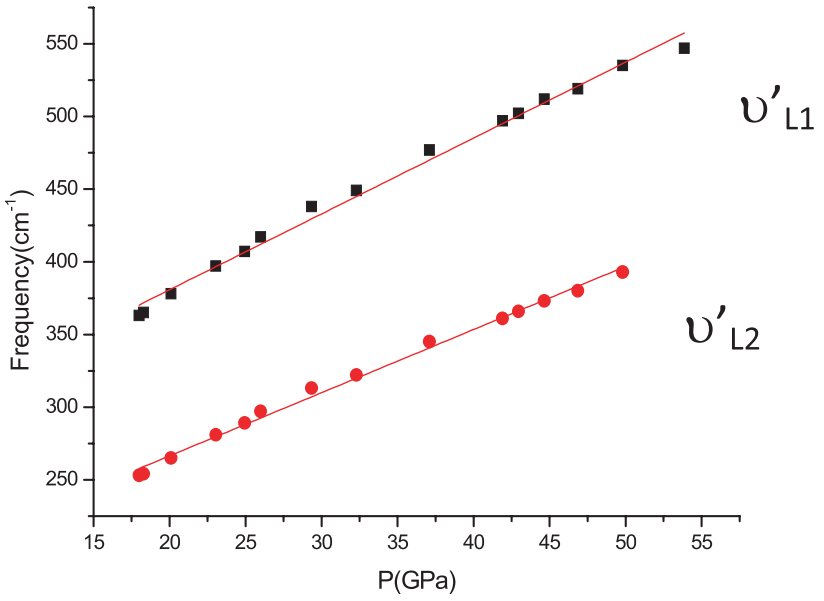

Fig. 1: The experimental Raman frequencies of the lattice modes $v_{L 1}^{\prime}$ and $v_{L_{2}}^{\prime}$ [2] as a function of pressure in the $\eta$ phase of solid $N_{2}$. Solid lines represent Eq. (1) fitted to the experimental data with the coefficients $v_{0}$ and $c$ (Table 1 ).

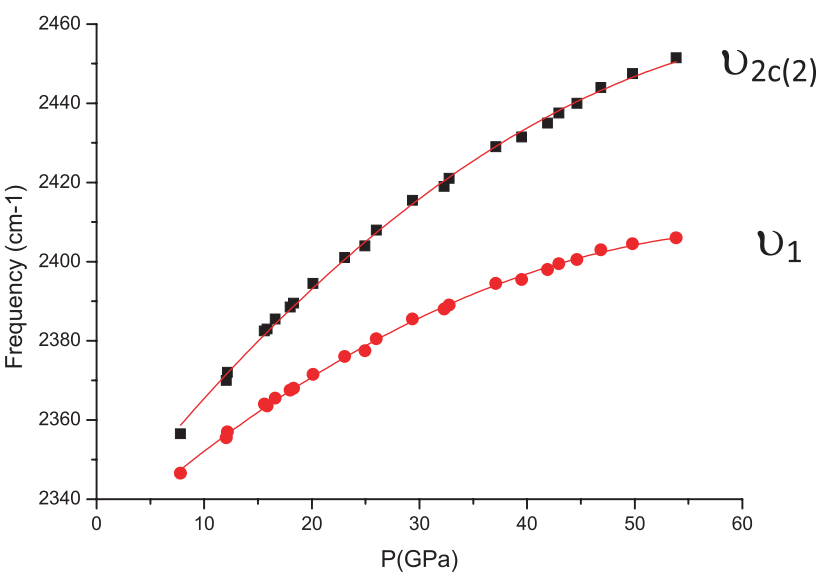

Fig. 2: The experimental Raman frequencies of the internal modes $v_{1}$ and $\mathrm{v}_{2 \mathrm{C}(2)}[2]$ as a function of pressure in the $\eta$ phase of solid $\mathrm{N}_{2}$. Solid lines represent Eq. (2) fitted to the experimental data with the coefficients $\mathrm{v}_{0}$, $\mathrm{C}$ and $\mathrm{c}^{\prime}$ (Table 1 ).

As the Raman frequency shifts, the mode Grüneisen parameters of the $\mathrm{N}_{2}$ lattice modes and vibrons are also pressure dependent, which have been determined from the experimental measurements in the $\eta$ phase of solid 


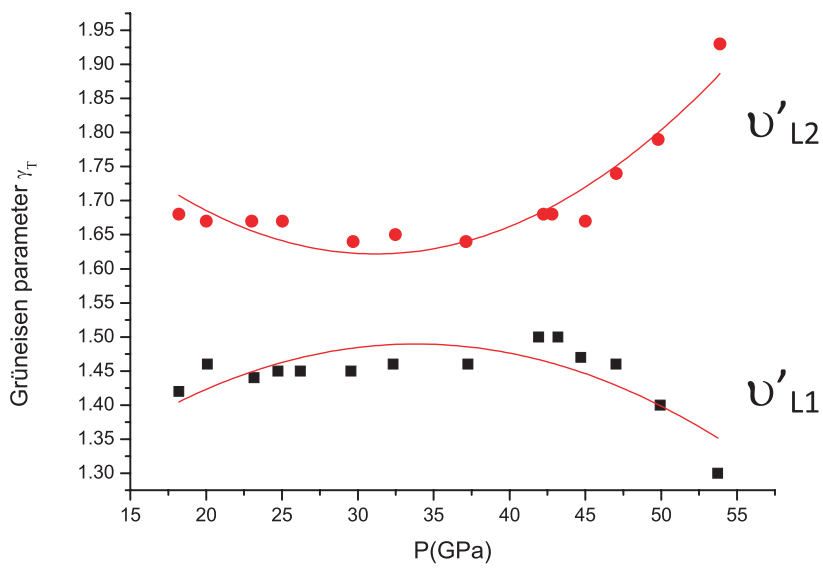

Fig. 3: Grüneisen parameter $\gamma_{T}$ of the lattice modes of $v_{{ }^{\prime} 1}^{\prime}$ and $v_{L 2}^{\prime}$ as a function of pressure for solid $\mathrm{N}_{2}$ [2]. Solid curves represent Eq. (3) fitted to the experimental data [2] with the coefficients $c_{0}, c_{1}$ and $c_{2}$ (Table 1).

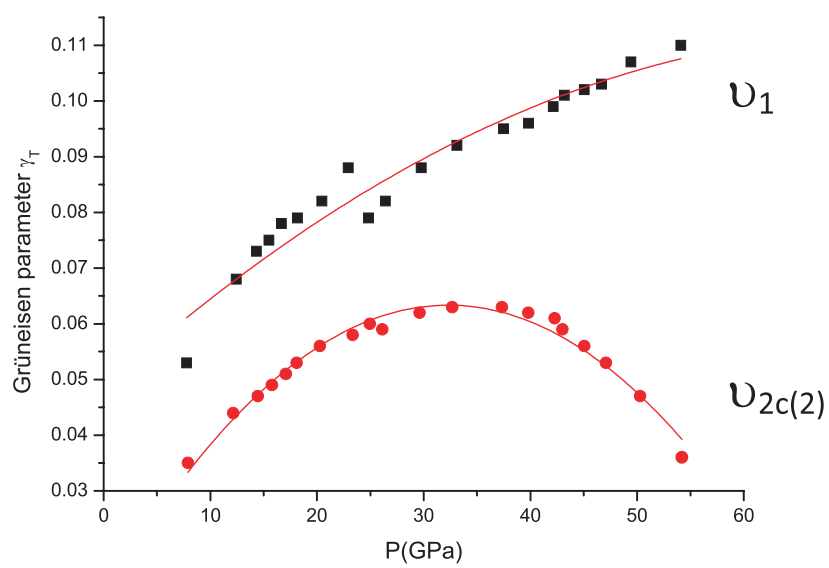

Fig. 4: Grüneisen parameter $\gamma_{T}$ of the $\mathrm{N}_{2}$ vibrons $\left(v_{1}\right.$ and $\left.v_{2 C(2)}\right)$ as a function of pressure for solid $\mathrm{N}_{2}$ [2]. Solid curves represent Eq. (3) fitted to the data [2] with the coefficients $c_{0}, c_{1}$ and $c_{2}$ (Table 1).

nitrogen [2]. The pressure dependence of the isothermal mode Grüneisen parameter $\gamma_{\mathrm{T}}$ can be expressed as

$$
\gamma_{\mathrm{T}}=\mathrm{c}_{0}+\mathrm{c}_{1} \mathrm{P}+\mathrm{c}_{2} \mathrm{P}^{2}
$$

where $\mathrm{c}_{0}, \mathrm{c}_{1}$ and $\mathrm{c}_{2}$ are constants. Eq. (3) was also fitted to the experimental data [2] given for the $\mathrm{N}_{2}$ lattice and vibrons, as plotted in Figs. 3 and 4, respectively. The coefficients $c_{0}, c_{1}$ and $c_{2}$ were determined for these Raman modes, which we give in Table 1.

By means of the mode Grüneisen parameter, the pressure dependence of the isothermal compressibility $\kappa_{\mathrm{T}}$ can be predicted according to the relation

$$
\gamma_{\mathrm{T}}=\left(1 / \kappa_{\mathrm{T}}\right)(1 / \mathrm{v})(\partial v / \partial P)_{\mathrm{T}}
$$

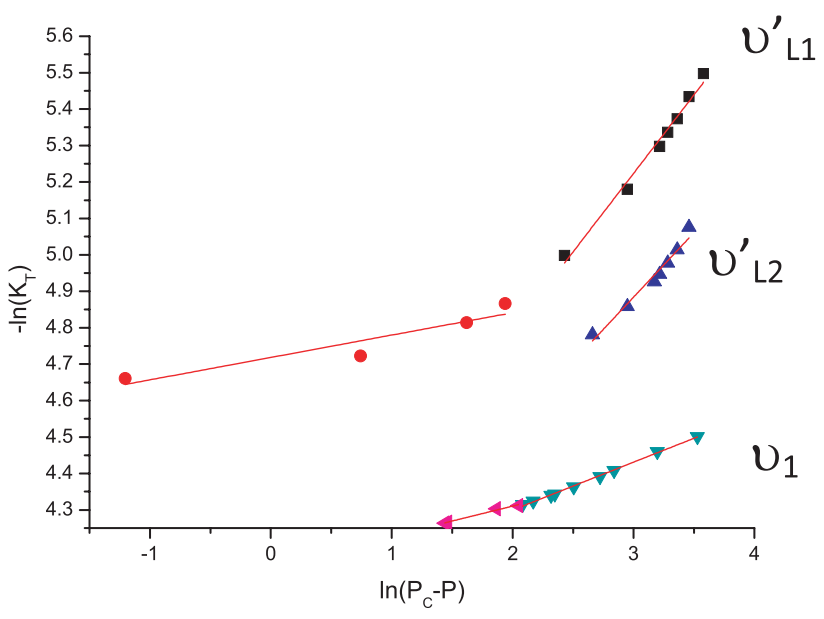

Fig. 5: The isothermal compressibility $\kappa_{T}$ in a log-log scale as a function of pressure in the $\eta$ phase close to the $\delta$ phase $\left(\mathrm{P}_{\mathrm{c}}=18 \mathrm{GPa}\right)$ of solid $\mathrm{N}_{2}$ at $300 \mathrm{~K}$ according to Eq. (5). The $\kappa_{\mathrm{T}}$ values were calculated using the frequency shifts of the lattice modes $v_{{ }_{L 1}}^{\prime}$ and $v_{L 2}^{\prime}$ and the internal mode $v_{1}$ (Eq. 4) with the values of the critical exponent $\gamma$ and the amplitude $\mathrm{A}$ (Table 2).

Thus, using the pressure dependence of the Raman frequency shifts for the $\mathrm{N}_{2}$ lattice modes and vibrons with their mode Grüneisen parameters, the isothermal compressibility $\kappa_{\mathrm{T}}$ was predicted as a function of pressure $(\mathrm{T}=300 \mathrm{~K})$ in the $\eta$ phase of solid nitrogen. This prediction of the $\kappa_{\mathrm{T}}$ was made close to the $\eta-\delta$ transition as the pressure varies $(\mathrm{T}=300 \mathrm{~K})$ around the critical pressure $\left(\mathrm{P}_{\mathrm{c}}=18 \mathrm{GPa}\right)[2]$ according to a power-law formula,

$$
\mathrm{K}_{\mathrm{T}}=\mathrm{A}\left(\mathrm{P}-\mathrm{P}_{\mathrm{c}}\right)^{-\gamma}
$$

where $\gamma$ is the critical exponent for the isothermal compressibility and $A$ is the amplitude. In Fig. 5 , the $\kappa_{T}$ is plotted as a function of $\mathrm{P}-\mathrm{P}_{\mathrm{c}}$ in a log-log scale according to Eq. (5), which was predicted using the Raman frequency shifts of the lattice modes $\left(v_{\mathrm{L} 1}^{\prime}\right.$ and $\left.v_{\mathrm{L} 2}^{\prime}\right)$ and the $\mathrm{N}_{2}$ vibron $\left(v_{1}\right)$ (Eq. 4). The critical behavior of the isothermal compressibility $\kappa_{\mathrm{T}}$ was determined in the two pressure intervals (Table 2), which we plot as straight lines in Fig. 5. Table 2 gives the values of the critical exponent $\gamma$ for the isothermal compressibility $\kappa_{\mathrm{T}}$ and the amplitude A for the pressure intervals indicated. The Raman modes whose frequency shifts were used for the critical behavior of the isothermal compressibility $\kappa_{\mathrm{T}}$, are also indicated in this table. From our study, the isothermal compressibility $\kappa_{\mathrm{T}}$ calculated from the Raman frequency shifts of $v_{2(2)}$ was not adequate as a function of pressure. 


\begin{tabular}{lllclrr} 
Raman modes & $\gamma$ & $\mathbf{A}$ & $\begin{array}{l}\text { Pressure interval } \\
\mathbf{P}-\mathbf{P}_{\mathrm{C}}(\mathrm{GPa})\end{array}$ & $\boldsymbol{A}$ & $\begin{array}{l}\text { Pressure interval } \\
\mathbf{P}-\mathbf{P}_{\mathrm{C}}(\mathbf{G P a})\end{array}$ \\
\hline $\mathrm{U}_{\mathrm{L} 1}^{\prime}$ & 0.16 & 0.010 & $0<\mathrm{P}-\mathrm{P}_{\mathrm{C}}<12$ & 0.43 & 0.020 & $11<\mathrm{P}-\mathrm{P}_{\mathrm{C}}<36$ \\
$\mathrm{U}_{\mathrm{L} 2}$ & 0.35 & 0.022 & $14<\mathrm{P}-\mathrm{P}_{\mathrm{C}}<32$ & 0.41 & 0.027 & $19<\mathrm{P}-\mathrm{P}_{\mathrm{C}}<32$ \\
$\mathrm{U}_{1}$ & 0.06 & 0.015 & $0<\mathrm{P}-\mathrm{P}_{\mathrm{C}}<6.5$ & 0.13 & 0.018 & $7<\mathrm{P}-\mathrm{P}_{\mathrm{C}}<34$ \\
$\mathrm{U}_{2 \mathrm{C}(2)}$ & 0.21 & $7.26 \times 10^{-6}$ & $0<\mathrm{P}-\mathrm{P}_{\mathrm{C}}<15.5$ & 2.05 & 0.018 & $42<\mathrm{P}-\mathrm{P}_{\mathrm{C}}<46$ \\
\hline
\end{tabular}

Table 2: Values of the critical exponent $\gamma$ for the isothermal compressibility $\kappa_{\mathrm{T}}$ and the amplitude $A$ in the pressure intervals according to Eq. (5) using the observed [2] frequency shifts of the Raman modes indicated at various pressures close to the $\eta-\delta$ transition in the solid $N_{2}$.

\subsection{Spectroscopic modification of the Pippard relations for the $\eta$ phase of $\mathrm{N}_{2}$}

Spectroscopic modifications of the Pippard relations can be obtained by relating the frequency shifts of the Raman modes studied to the thermodynamic quantities for the $\eta$ phase of solid nitrogen. Using the Pippard relations [21]

$$
\mathrm{C}_{\mathrm{P}}=\mathrm{T}(\mathrm{dP} / \mathrm{dT}) \mathrm{V} \alpha_{\mathrm{P}}+\mathrm{T}(\mathrm{dS} / \mathrm{dT})
$$

and

$$
\alpha_{\mathrm{P}}=(\mathrm{dP} / \mathrm{dT}) \kappa_{\mathrm{T}}+(1 / \mathrm{V})(\mathrm{dV} / \mathrm{dT})
$$

the frequency shifts $(\partial v / \partial P)$ can be related to the thermal expansion $\alpha_{\mathrm{p}}$. Eq. (7) can be rewritten through the isothermal mode Grüneisen parameter $\gamma_{\mathrm{T}}$ (Eq. 4) as

$$
\alpha_{\mathrm{P}}=\left(1 / \gamma_{\mathrm{T}}\right)(\mathrm{dP} / \mathrm{dT})(1 / v)(\partial v / \partial \mathrm{P})+(1 / \mathrm{V})(\mathrm{dV} / \mathrm{dT})
$$

Thus, a linear variation of $\alpha_{\mathrm{p}}$ with the $(1 / v)(\partial v / \partial \mathrm{P})_{\mathrm{T}}$ can be obtained by calculating the thermal expansion $\alpha_{\mathrm{p}}$ from the isothermal compressibility $\kappa_{\mathrm{T}}$ according to the thermodynamic relation

$$
\alpha_{\mathrm{P}} / \kappa_{\mathrm{T}}=\mathrm{dP} / \mathrm{dT}
$$

Since we obtained the pressure dependence of the isothermal compressibility $\kappa_{\mathrm{T}}$ close to the $\eta-\delta$ transition using Eq. (5) where the critical exponent $\gamma$ and the amplitude A were determined (Table 2), the pressure dependence of the thermal expansion $\alpha_{\mathrm{p}}$ was then determined according to Eq. (9). For this determination, the slope $\mathrm{dP} / \mathrm{dT}$ was extracted from the experimental data [2]. By extending the experimental phase line between the $\epsilon$ and $\delta$ phases close to the phase $\eta$, to the room temperature $(\mathrm{T}=300 \mathrm{~K}) \mathrm{ac}$ cording to a linear relation

$$
\mathrm{P}=\mathrm{a}_{0}+\mathrm{a}_{1} \mathrm{~T}
$$

\begin{tabular}{llll} 
Phases & $-\mathrm{a}_{0}(\mathrm{GPa})$ & $\mathrm{a}_{1}(\mathrm{GPa} / \mathrm{K})$ & $\mathrm{dP} / \mathrm{dT}(\mathrm{GPa} / \mathrm{K})$ \\
\hline$\eta-\delta$ & 2.21 & 0.065 & 0.065 \\
\hline
\end{tabular}

Table 3: $V$ alues of the coefficients $a_{0}$ and $a_{1}$ according to Eq. (10) which was fitted to the experimental $\eta-\delta$ phase line [2] in the solid $\mathrm{N}_{2}$.

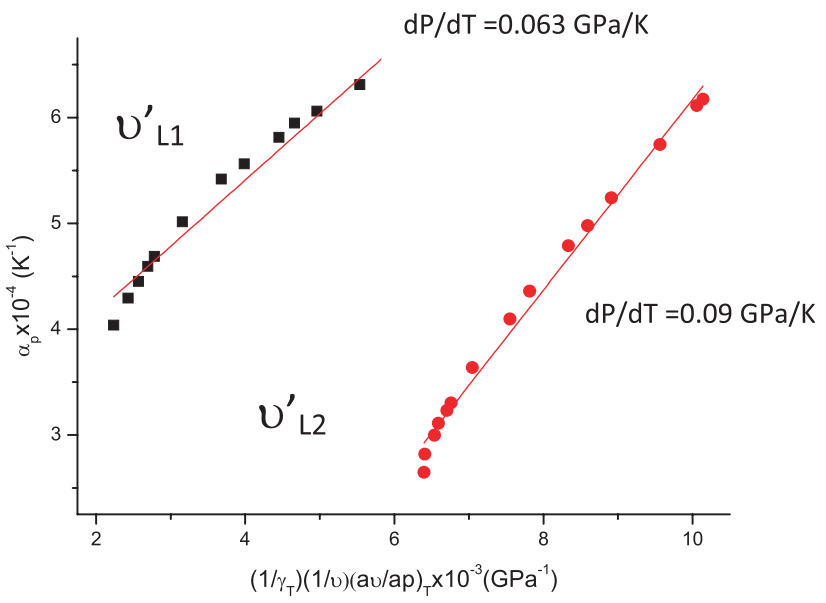

Fig. 6: Thermal expansion $\alpha_{p}$ as a function of the frequency shifts of the lattice modes $\left(v^{\prime}{ }_{11}\right.$ and $\left.v^{\prime}{ }_{L 2}\right)$ according to Eq. (8) for the $\eta$ phase of solid $\mathrm{N}_{2}$.

where $\mathrm{a}_{0}$ and $\mathrm{a}_{1}$ are constants, the slope $\mathrm{dP} / \mathrm{dT}\left(=\mathrm{a}_{1}\right)$ was obtained, as given in Table 3. Using this slope value and the pressure dependence of the isothermal compressibility (Eq. 5) which was obtained from the frequency shifts of the Raman modes, as given in Fig. 5, values of the thermal expansion $\alpha_{p}$ were calculated (Eq. 9) as a function of pressure $(\mathrm{T}=300 \mathrm{~K})$ close to the $\eta$ phase of solid nitrogen. Figs. 6 and 7 give our plots of $\alpha_{p}$ as a function of $\left(1 / \gamma_{T}\right)(1 / v)$ $(\partial v / \partial P)_{\mathrm{T}}$ for the lattice modes $\left(v_{\mathrm{L} 1}^{\prime}\right.$ and $\left.v_{\mathrm{L} 2}^{\prime}\right)$ and for the internal modes $\left(v_{1}\right.$ and $\left.v_{2 c(2)}\right)$, respectively, according to the spectroscopic modification of the Pippard relation (Eq. 8). The values of the slope $\mathrm{dP} / \mathrm{dT}$ and the intercept $(1 / \mathrm{V})(\mathrm{dV} /$ dT) which we extracted from the Raman modes studied, are given in Table 4. 


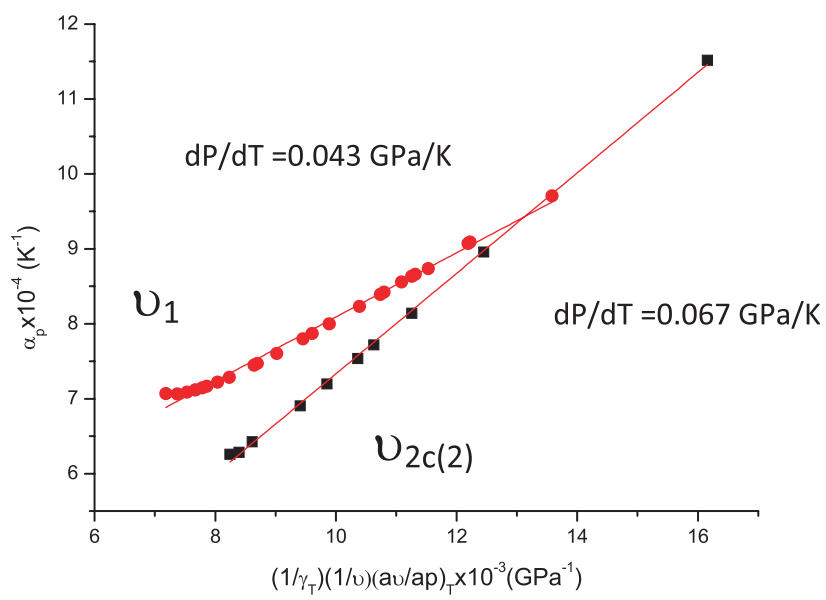

Fig. 7: Thermal expansion $\alpha_{p}$ as a function of the frequency shifts of the internal modes $\left(v_{1}\right.$ and $\left.v_{2 c(2)}\right)$ according to Eq. (8) for the $\eta$ phase of solid $\mathrm{N}_{2}$.

\begin{tabular}{llll}
$\begin{array}{l}\text { Raman } \\
\text { modes }\end{array}$ & $\begin{array}{l}\mathrm{dP} / \mathrm{dT} \\
(\mathrm{GPa} / \mathrm{K})\end{array}$ & $\begin{array}{l}(\mathbf{1} / \mathrm{V})(\mathrm{dV} / \mathrm{dT}) \\
\left(\mathrm{K}^{-1}\right)\end{array}$ & $\begin{array}{l}\mathrm{dP} / \mathbf{d T} \\
(\mathrm{GPa} / \mathrm{K})\end{array}$ \\
\hline $\mathrm{U}_{L 1}^{\prime}$ & 0.063 & 3.0 & 0.065 \\
$\mathrm{U}_{L 2}^{\prime}$ & 0.096 & 4.0 & 0.065 \\
$\mathrm{U}_{1}$ & 0.043 & 4.0 & 0.065 \\
$\mathrm{U}_{2 \mathrm{C}(2)}$ & 0.067 & 0.7 & 0.065 \\
\hline
\end{tabular}

Table 4: Values of the slope $\mathrm{dP} / \mathrm{dT}$ and the intercept $(1 / \mathrm{V})(\mathrm{dV} / \mathrm{dT})$ according to Eqs. (7) and (8) for the Raman modes indicated in the $\eta$ phase of solid $\mathrm{N}_{2}$. dP/dT values $\eta$ extracted from Eq. (11) through Eq. (8) for the Raman modes are also given.

The pressure dependence of the specific heat was also calculated in this study according to the thermodynamic relation

$$
\mathrm{C}_{\mathrm{P}}-\mathrm{C}_{\mathrm{v}}=\mathrm{TV} \alpha_{\mathrm{p}}^{2} / \kappa_{\mathrm{T}}
$$

for the $\eta$ phase of solid nitrogen. Using the pressure dependence of $\alpha_{\mathrm{P}}$ and $\kappa_{\mathrm{T}}$ at $\mathrm{T}=300 \mathrm{~K},\left(\mathrm{C}_{\mathrm{P}}-\mathrm{C}_{\mathrm{v}}\right) / \mathrm{V}$ was obtained at various pressures in the $\eta$ phase of solid nitrogen. Since the values of $\alpha_{\mathrm{P}}$ and $\kappa_{\mathrm{T}}$ were obtained using the frequency shifts of the Raman modes $\left(v_{L_{1}}^{\prime}\right.$ and $v_{L_{2}}^{\prime}, v_{1}$ and $\left.\mathrm{v}_{2 \mathrm{c}(2)}\right)$, they were used to calculate $\left(\mathrm{C}_{\mathrm{P}}-\mathrm{C}_{\mathrm{v}}\right) / \mathrm{V}$ as a function of pressure $(\mathrm{T}=300 \mathrm{~K}) .\left(\mathrm{C}_{\mathrm{p}}-\mathrm{C}_{\mathrm{v}}\right) / \mathrm{V}$ values are plotted as a function of $\alpha_{\mathrm{p}}$ using the frequency shifts of the lattice modes $\left(v_{L 1}^{\prime}\right.$ and $\left.v_{L 2}^{\prime}\right)$ and the internal modes $\left(v_{1}\right.$ and $\left.v_{2 c(2)}\right)$, respectively, in Figs. 8 and 9 according to the Pippard relation (Eq. 6) where $\mathrm{C}_{\mathrm{v}}=\mathrm{T}(\mathrm{dS} / \mathrm{dT})$. The experimental value of the slope, $\mathrm{dP} / \mathrm{dT}=0.065 \mathrm{GPa} / \mathrm{K}$ (Table 3) was obtained from both plots (Table 4) which was used initially as also shown in Figs. 8 and 9.

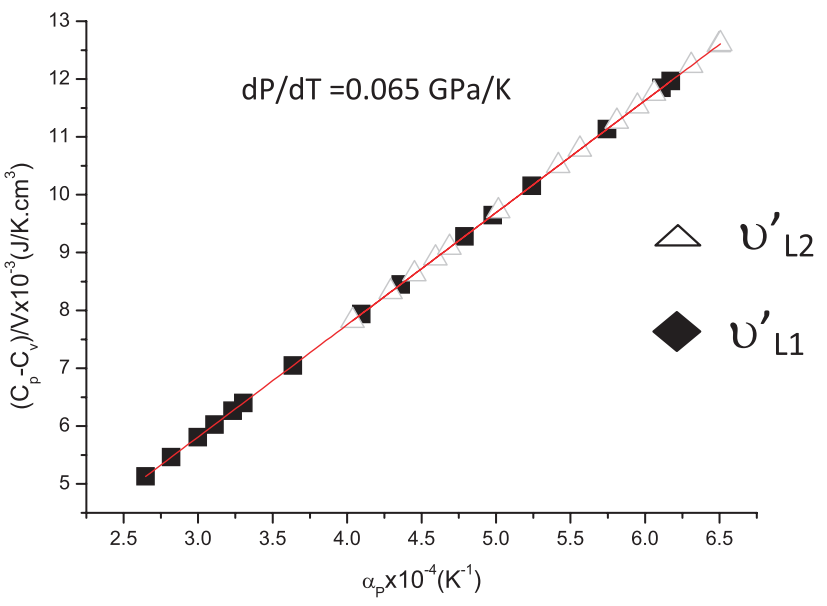

Fig. 8: The difference in the specific heat per unit volume as a function of the thermal expansion $\alpha_{p}$ according to Eq. (6) using the Raman frequencies of the $v_{{ }_{1}}^{\prime}$ and $v^{\prime}{ }_{22}$ modes of solid $N_{2}$.

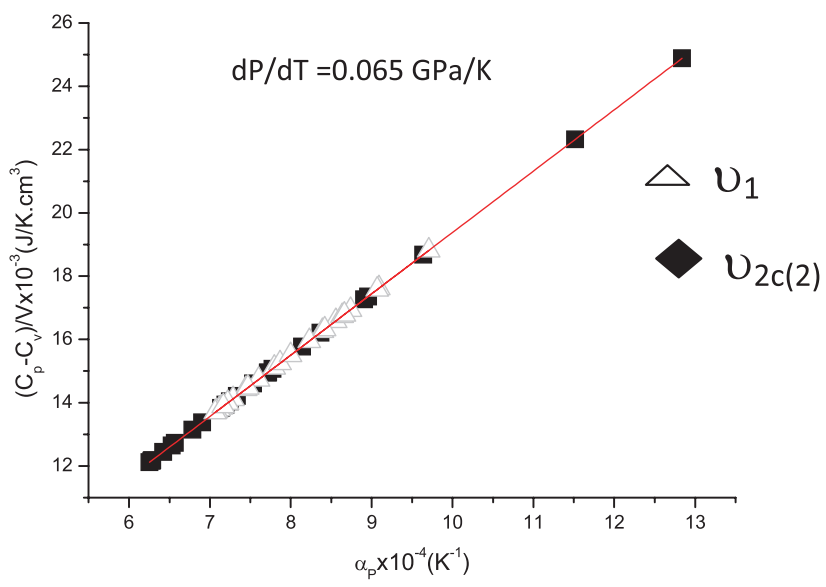

Fig. 9: The difference in the specific heat per unit volume as a function of the thermal expansion $\alpha_{p}$ according to Eq. (6) using the Raman frequencies of the $v_{1}$ and $v_{2 c(2)}$ modes for the $\eta$ phase of solid $\mathrm{N}_{2}$.

\section{Discussion}

The Raman frequency shifts of the $\mathrm{N}_{2}$ lattice modes $\left(\mathrm{v}_{\mathrm{L} 1}^{\prime}\right.$ and $\left.v_{\mathrm{L} 2}^{\prime}\right)$ and vibrons $\left(v_{1}\right.$ and $\left.v_{2 c(2)}\right)$ were related to the thermodynamic functions of the isothermal compressibility $\kappa_{T}$, thermal expansion $\alpha_{P}$ and the specific heat $\left(C_{P}-C_{v}\right)$ for the $\eta$ phase of solid nitrogen. These correlations were obtained by analyzing the Raman frequencies considered according to Eqs. (1) and (2), as given in Figs. 1 and 2, respectively (Table 1$)$. The pressure dependence of the Raman frequencies was represented by linear (Eq. 1) and quadratic (Eq. 2) relations using the experimental data, as shown in the figures. The Raman frequencies of the modes studied increase as the pressure increases as obtained 
experimentally (Figs. 1 and 2). However, the pressure dependence of the mode Grüneisen parameters for the Raman modes except the $v_{1}$ mode (Fig. 4) is completely different, as shown in Figs. 3 and 4. The $\gamma_{\mathrm{T}}$ values increase as the pressure increases up to $55 \mathrm{GPa}$ for the $\mathrm{v}_{1}$ mode, whereas for the $v_{2 \mathrm{c}(2)}$ mode the $\gamma_{\mathrm{T}}$ increases with the pressure to about $30 \mathrm{GPa}$, then it decreases with increasing pressure (Fig. 4). Regarding the $v_{\mathrm{L} 1}^{\prime}$ and $v_{\mathrm{L} 2}^{\prime}$ modes (Fig. 3), the pressure dependence of the mode Grüneisen parameter $\gamma_{T}$ is not very well represented by a quadratic function (Eq. 3) because of the scattered experimental data [2] for both lattice modes (Fig. 3), although the quadratic function is the best suited to the data available.

In this study, we also analyzed the critical behavior of the isothermal compressibility $\kappa_{\mathrm{T}}$ which was calculated using the Raman frequency shifts of the Raman modes through the mode Grüneisen parameter $\gamma_{\mathrm{T}}$ (Eq. 4). From the pressure dependence of the frequency shifts of both $\mathrm{N}_{2}$ lattice modes and vibrons, values of the critical exponent $\gamma$ for the $\kappa_{\mathrm{T}}$ were extracted (Table 2) which increase as the pressure interval increases with respect to the critical pressure $P_{c}$. For the lattice modes of $v_{L 1}^{\prime}$ and $v_{L 2}^{\prime}$ and the internal mode $v_{1}$, this increase in the $\gamma$ value is reasonable, but the value of $\gamma=2.05$ due to the $v_{2 \mathrm{c}(2)}$ mode close to the $\mathrm{P}_{\mathrm{c}}$ (Table 2) is too large to describe the critical behavior of $\kappa_{\mathrm{T}}$ in the $\eta$ phase of solid nitrogen.

We finally constructed the Pippard relations here according to Eqs. (6) and (8) using the pressure dependence of the Raman modes studied for the $\eta$ phase of solid $N_{2}$. As shown in Figs. 6-9, a linear variation of $\alpha_{\mathrm{p}}$ with the $(1 / v)(\partial v / \partial \mathrm{P})_{\mathrm{T}}$ and also a linear variation of $\left(\mathrm{C}_{\mathrm{P}}-\mathrm{C}_{\mathrm{V}}\right) / \mathrm{V}$ with the $\alpha_{\mathrm{P}}$ were obtained. The slope value of $\mathrm{dP} / \mathrm{dT}=0.065$ $\mathrm{GPa} / \mathrm{K}$ was deduced from our linear plots (Figs. 8 and 9), which is the same value obtained experimentally (Table $3)$. In the case of $\alpha_{\mathrm{P}}$ plotted as a function of $(1 / \mathrm{v})(\partial \mathrm{v} / \partial \mathrm{P})_{\mathrm{T}}$ for the lattice modes of $v_{L 1}^{\prime}$ and $v_{L 2}^{\prime}$ (Fig. 6), values of the slope $\mathrm{dP} / \mathrm{dT}$ were deviated from the experimental value of $0.065 \mathrm{GPa} / \mathrm{K}$. Since we calculated the pressure dependence of the specific heat $\left(\mathrm{C}_{\mathrm{P}}-\mathrm{C}_{\mathrm{V}}\right)$ from Eq. (11) where the slope value of $\mathrm{dP} / \mathrm{dT}=0.065 \mathrm{GPa} / \mathrm{K}$ was used initially as the experimental value (Table 3 ), the same slope value should be extracted from a plot of $\left(\mathrm{C}_{\mathrm{P}}-\mathrm{C}_{\mathrm{V}}\right) / \mathrm{V}$ vs. $\alpha_{\mathrm{P}}$ using the Raman frequency shifts of both $\mathrm{N}_{2}$ lattice modes (Fig. 8). On the other hand, the slope value of $\mathrm{dP} / \mathrm{dT}$ deduced from the Raman frequency shifts $(1 / v)(\partial v / \partial \mathrm{P})_{\mathrm{T}}$ for the lattice modes of $v_{\mathrm{L} 1}^{\prime}$ and $v_{\mathrm{L} 2}^{\prime}$, as plotted in Fig. 6, is determined by the pressure dependence of the Raman frequencies (Fig. 1) and also of the mode Grüneisen parameter $\gamma_{\mathrm{T}}$ (Fig. 3). In the pressure interval studied, variation of the Raman frequencies of the $v_{\mathrm{L} 1}^{\prime}$ and $v_{\mathrm{L} 2}^{\prime}$ modes (Fig. 1) with the pressure is well represented by Eq. (1). However, the mode
Grüneisen parameter $\gamma_{T}$ is not well represented by a quadratic function due to the scattered experimental data, as stated above. This may cause a nonlinear behavior of our plots of $\alpha_{\mathrm{P}} \mathrm{Vs} .(1 / \mathrm{v})(\partial \mathrm{v} / \partial \mathrm{P})_{\mathrm{T}}$ for the lattice modes of $\mathrm{v}_{\mathrm{L} 1}^{\prime}$ and $v_{L 2}^{\prime}$ (Fig. 6) in the $\eta$ phase of solid nitrogen according to Eq. (8). So, the slope $\mathrm{dP} / \mathrm{dT}$ changes for this nonlinear behavior (Fig. 6) if we choose, say, two different pressure intervals in the $\eta$ phase of solid $N_{2}$. As plotted in Fig. 7, the spectroscopic modification of the Pippard relation (Eq. 8) was obtained from a linear variation of the $\alpha_{p}$ with the frequency shifts $(1 / v)(\partial v / \partial \mathrm{P})_{\mathrm{T}}$ for both the internal modes of $v_{1}$ and $v_{2(2)}$. This linear variation deviates as the frequency shifts decrease for the $v_{1}$ mode (Fig. 7), whereas for the $v_{2 c(2)}$ mode this linearity is better represented by Eq. (8). This linear variation of $\alpha_{\mathrm{p}}$ with the $(1 / v)(\partial v / \partial P)_{\mathrm{T}}$ for the $v_{2 c(2)}$ mode (Fig. 7 ) gives rise to the experimentally obtained value between the phases $\eta$ and $\delta$ in solid $\mathrm{N}_{2}$ (Tables 3 and 4). On the other hand, the $\mathrm{dP} / \mathrm{dT}$ value extracted from the Raman frequency shifts of the $v_{2 c(2)}$ mode is not too far away from the experimental value of $0.065 \mathrm{GPa} / \mathrm{K}$ (Table 4). When we plotted $\left(C_{P}-C_{v}\right) / V$ vs. $\alpha_{p}$ using the Raman frequency shifts of the $v_{1}$ and $v_{2 c(2)}$ modes, as given in Fig. 9, linear variations were obtained according to Eq. (6), from which the slope value of $\mathrm{dP} / \mathrm{dT}=0.065 \mathrm{GPa} / \mathrm{K}$ (Table 4) was extracted, as for the lattice modes of $v_{L 1}^{\prime}$ and $v_{L 2}^{\prime}$. This slope value is the same as the experimental $\mathrm{dP} / \mathrm{dT}$ value (Table 3). A linear variation of $\left(\mathrm{C}_{\mathrm{P}}-\mathrm{C}_{\mathrm{v}}\right) / \mathrm{V}$ vs. $\alpha_{\mathrm{p}}$ (Figs. 8 and 9) and the $\mathrm{dP} / \mathrm{dT}$ values deduced, also validates the Pippard relation (Eq. 6) through the spectroscopic modification of the Pippard relation (Eq. 8) for the $\mathrm{N}_{2}$ lattice modes and vibrons in the $\eta$ phase of solid nitrogen.

Finally, regarding the quadratic pressure dependence of the isothermal mode Grüneisen parameter (Eq. 3) we deduced the $\gamma_{T}$ values of the lattice modes at zero pressure $(\mathrm{P}=0)$ with the coefficient $\mathrm{c}_{0}$ (Table 1) as $1.1\left(\mathrm{v}_{\mathrm{L} 1}^{\prime}\right)$ and 2.1 $\left(v_{L 2}^{\prime}\right)$. Our value of $\gamma_{T}=1.1$ for the $v_{L 1}^{\prime}$ is close to the value of 5/6 expected for a quadrupolar interaction potential using the quasi-harmonic approximation and it is also in the range of $1<\gamma \leq 1.5$ due to nuclear quadrupole resonance methods [22]. Our $\gamma_{T}$ values for the internal modes $\left(v_{1}\right.$ and $\left.v_{2 c(2)}\right)$ are too small to be compared with the model expectations.

\section{Conclusions}

The experimental Raman frequency shifts and the mode Grüneisen parameters were analyzed for the $\mathrm{N}_{2}$ vibrons at various high pressures $(\mathrm{T}=300 \mathrm{~K})$ in the $\eta$ phase of solid nitrogen. The critical behavior of the isothermal compressibility calculated from the Raman frequency shifts 
was described by the critical exponent close to the $\eta-\delta$ transition. By calculating the pressure dependence of the thermal expansion and the specific heat, the Pippard relations (and their spectroscopic modification) were established in the $\eta$ phase of solid nitrogen.

Our results show that the thermodynamic quantities can be calculated from the Raman frequency shifts and that the Pippard relations can be verified for the $\eta$ phase of solid nitrogen.

Received: October 19, 2012. Accepted: November 30, 2012.

\section{References}

[1] R. Reichlih, D. Schferl, S. Martin, C. Vanderborgh and R.L. Mills, Phys. Rev. Lett. 55 (1985) 1464.

[2] H. Schneider, W. Hafner, A. Wokaun and H. Olijnyk, J. Chem. Phys. 96 (1992) 8046.

[3] M.M. Scheerboom and J.A. Schouten, Phys. Rev. Lett. 71 (1993) 2252.

[4] T. Westerhoff, A. Wittig and R. Feile, Phys. Rev. B54 (1996) 14.

[5] E. Gregoryanz, A.F. Goncharov, R.J. Hemley, H.K. Mao, M. Somayazulu and G. Shen, Phys. Rev. B66 (2002) 224108.

[6] M. Grimsditch, Phys. Rev. B32 (1985) 514.
[7] R.L. Mills, B. Olinger and D.T. Cromer, J. Chem. Phys. 84 (1986) 2837.

[8] D. Schiferl, R. Lesar and D.S. Moore, in The Simple Molecular Systems at Very High Density (Les Houches, France, 1988), edited by A. Polian, P. Loubeyre and N. Boccara (Plenum, New York, 1989), p. 303.

[9] H. Olijnyk, J. Chem. Phys. 93 (1990) 8968.

[10] A. Anderson, T.S. Sun and M.C.A. Donkersloot, Can. J. Phys. 48 (1970) 2265.

[11] M.M. Thiery, D. Fabre, M. Jean-Louis and H. Vu, J. Chem. Phys. 59 (1973) 4559.

[12] F.D. Medina and W.B. Daniels, J. Chem. Phys. 64 (1976) 150.

[13] H. Yurtseven and T. Tunay, Int. J. Mod. Phys. 24 (2010) 6069.

[14] T.S. Kuan, A. Warshel and O. Schnepp, J. Chem. Phys. 52 (1970) 3012.

[15] T. Luty and G.S. Pawley, Chem. Phys. Lett. 28 (1974) 593.

[16] M.J. Mandell, J. Low Temp. Phys. 17 (1974) 169.

[17] A. Zunger and E. Huler, J. Chem. Phys. 62 (1975) 3010.

[18] V.G. Manzhelii, A.M. Tolkachev and E.I. Vortovich, Phys. Stat. Sol. 13 (1966) 351.

[19] D.C. Heberlein, E.D. Adams and T.A. Scott, J. Low Temp. Phys. 2 (1970) 449.

[20] M.I. Bagatskii, V.A. Kutcheryavy, M.G. Manzhalii and V.A. Popov, Phys. Stat. Sol. 26 (1968) 453.

[21] A.B. Pippard, The Flements of Classical Thermodynamics, Cambridge Univ. Press, New York, 1957.

[22] J.R. Brookeman, M.M. McFnan and T.A. Scott, Phys. Rev. B4 (1971) 3661. 
